\title{
Effect of open Mg sintering ambiance on the in-field critical current density of ex-situ $\mathrm{MgB}_{2}$
}

\author{
Bhavesh B. Sinha, S. H. Jang, and Kookchae Chung
}

Functional nano-materials research department, Korea Institute of Material Science, Changwon, South Korea

Received 2 October 2012; accepted 13 November 2012

\begin{abstract}
MgB}_{2}$ superconductor is highly sensitive to the Mg content. Even if the samples are synthesized with the appropriate looking stoichiometric ratio, the heat treatment leads to the loss of $\mathrm{Mg}$ either to ambiance or to MgO. To avoid it, either excess $\mathrm{Mg}$ is added in the starting powder or sealed ampoule annealing is employed. In this paper the effect of open $\mathrm{Mg}$ sintering ambiance on the ex-situ $\mathbf{M g B}_{2}$ was studied to enhance its superconducting properties. The open Mg ambiance was created to avoid any overpressure of $\mathrm{Mg}$ by providing a hole in Fe tube used as sample holder. The decrease in resistivity of the synthesized sample was observed through the increased temperature dependence of electron-phonon interactions. A clear enhancement in the superconducting cross-sectional area and hence the in-field critical current density is obtained.
\end{abstract}

Keywords: $\mathrm{MgB}_{2}$, sintering, stoichiometry, critical current density, flux pinning.

\section{INTRODUCTION}

$\mathrm{MgB}_{2}$ superconductor has been manifested to be highly sensitive to composition and the history of sample preparation. Since its discovery in 2001 [1], $\mathrm{MgB}_{2}$ has been examined in different forms through various processes of synthesis for superconducting properties [2$6]$.

$\mathrm{MgB}_{2}$ being a binary intermetallic compound, is considered easy to synthesize. But the vast difference in melting point of $\mathrm{B}$ and $\mathrm{Mg}$ makes the synthesis a bit tricky. Even if samples are synthesized, it shows widely different properties depending upon the synthesis and used precursor. A fantastic review can be found in [7] wherein resistivity of various samples prepared by different techniques of synthesis is compared. It is remarkable to see that the samples show $39 \mathrm{~K}$ superconducting transition temperature $\left(T_{\mathrm{c}}\right)$ even with the room temperature resistivity values of little over $100 \mathrm{~m} \Omega \mathrm{cm}$ [8] and also with less than $10 \mu \Omega \mathrm{cm}$ [9]. Different level of grain connectivity among the superconducting grains depending upon the extent of impurities, second phases and stoichiometry is anticipated as the reason for the varying superconducting parameters. Several studies had made it quite clear that unlike high $T_{\mathrm{c}}$ superconductors there persists a strong intergranular

*Corresponding author: kcchung@kims.re.kr current flow in $\mathrm{MgB}_{2}$ superconductor [10-12]. Leaving aside the defects and grain boundary effects, the improvement in critical current density of pure $\mathrm{MgB}_{2}$ is related with the improvement in the phase formation and increase in the sample density [13]. The main parameter behind the discrepancies in various $\mathrm{MgB}_{2}$ samples is the high vapor pressure of $\mathrm{Mg}$ which results in the instability of $\mathrm{Mg}$ content.

In the present investigation an interesting study to improve the superconducting properties of $\mathrm{MgB}_{2}$ superconductor merely by using $\mathrm{Mg}$ sintering ambiance is presented. The use of $\mathrm{MgB}_{2}$ powder as a precursor is anticipated towards nullifying the effect of preparative conditions in the synthesis of in-situ $\mathrm{MgB}_{2}$.

\section{EXPERIMENTALS}

A commercially available magnesium diboride from Alfa Aesar was used as a precursor powder. The aspurchased $\mathrm{MgB}_{2}$ powder with the particle size of 100 mesh and purity of $99 \%$ was hand milled in an agate mortar-pestle for about two hours in a glove box filled with ultrahigh pure Ar. This powder was then transferred in a mold to form a pellet of size $10 \mathrm{~mm} \mathrm{X} 10 \mathrm{~mm}$. The pressure used for making pellet was 10 ton $/ \mathrm{cm}^{2}$. Further these pellets were introduced into $\mathrm{Fe}$ tube with a very small hole on the lid to maintain inert environment. The samples were then heat treated in a tube furnace at $900^{\circ} \mathrm{C}$ for 3 hours. A continuous flow of ultra high pure Ar gas was maintained at 2 liters/min.

Two $\mathrm{MgB}_{2}$ samples were formed using the same process and the only difference in these two samples was the sintering environment. One sample was placed alone, while another sample was placed along with $\geq 99 \%$ pure $\mathrm{Mg}$ powder (Aldrich) in Fe tube. The amount of powder introduced was equal to the weight of pellet, in this case $157 \mathrm{mg}$ and was placed at a distance from pellet. The small hole in the Fe tube was purposely introduced to maintain an inert environment as well as to avoid any over pressure of $\mathrm{Mg}$ in the tube. It ensures self diffusion of $\mathrm{Mg}$ in pellet and excess $\mathrm{Mg}$ will easily pass out through the hole. Thus the overall $\mathrm{Mg}$ pressure inside the Fe tube was maintained same as ambient pressure. This will ensure an equilibrium diffusion of $\mathrm{Mg}$ in the sample and avoid any forceful $\mathrm{Mg}$ infiltration unlike in case of sealed tube. Hence the two types of samples were 


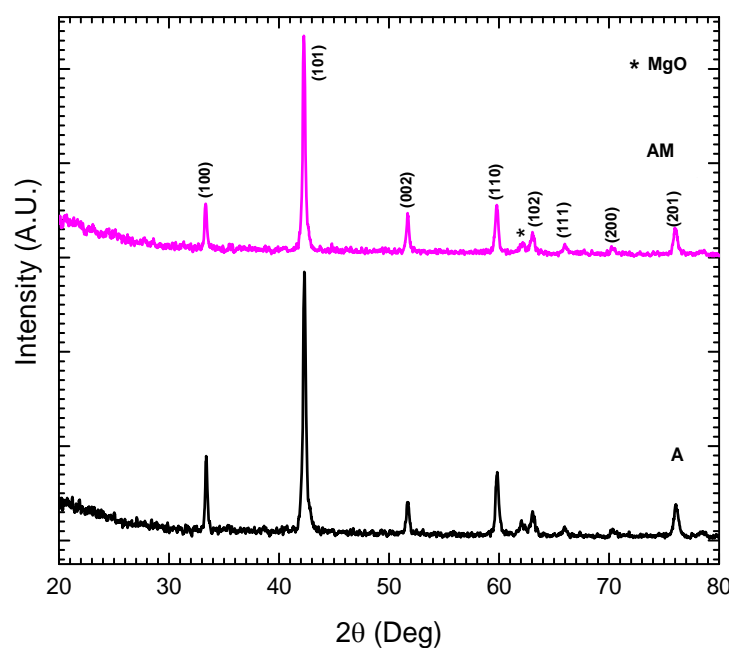

Fig. 1. X-ray diffraction patterns for A and AM samples.

prepared and named as A and AM based on the ambiance present during sintering.

The as-synthesized samples were then subjected to Xray diffraction (Rigaku D/Max 2200, $\mathrm{Cu} \mathrm{K \alpha}$ radiation) to study any variation in the $\mathrm{MgB}_{2}$ crystal structure. Superconducting transition temperature in the sample was studied by measuring resistivity by collinear four probe technique as well as zero-field-cooled and field-cooled magnetization measurements using Quantum Design physical property measurement system model no. 6000 . The magnetization against field measurements was carried out to determine irreversibility field. The critical current density was estimated by revoking the modified Bean's critical state model [14] as shown in equation below.

$$
\mathrm{J}_{\mathrm{c}}=\frac{20 \Delta \mathrm{M}}{\mathrm{a}\left(1-\frac{\mathrm{a}}{3 \mathrm{~b}}\right)}
$$

Where, $J_{\mathrm{c}}$ is critical current density in $\mathrm{A} / \mathrm{cm}^{2}, \Delta \mathrm{M}$ is the difference in the magnetization in emu $/ \mathrm{cm}^{3}$ for increasing and decreasing field, ' $a$ ' and ' $b$ ' is the thickness and width of samples.

\section{RESULTS AND DISCUSSION}

\subsection{X-ray diffraction studies}

Fig. 1 shows X-ray diffraction patterns (XRD) for the as-synthesized samples. Since $\mathrm{MgB}_{2}$ powder was used as a precursor, the XRD pattern shows all characteristic peaks corresponding to hexagonal crystal structure of $\mathrm{MgB}_{2}$ (JCPDS code: 38-1369). Small peak indicating presence of $\mathrm{MgO}$ is unavoidable. It is noteworthy to mention that XRD for sample AM do not show diffraction peaks of $\mathrm{Mg}$. This clarifies the absence of unreacted $\mathrm{Mg}$ in the sample even if it is sintered in $\mathrm{Mg}$ ambiance. This truly explains the Fe tube arrangement with a small hole to maintain appropriate $\mathrm{Mg}$ ambiance. The absence of $\mathrm{Mg}$ peaks implies that the $\mathrm{Mg}$ diffusing in the sample during sintering process is used up to satisfy any magnesium deficient secondary phases within the sample.

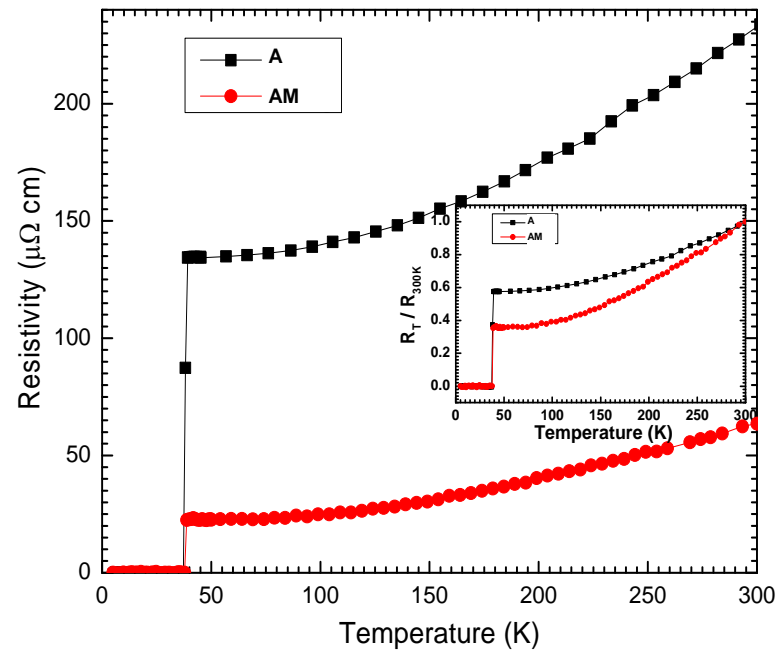

Fig. 2. Temperature-dependent resistivity plots for sample sintered with and without $\mathrm{Mg}$ ambiance. Inset shows the reduced resistivity plots.

\subsection{Resistivity Studies}

Even though excess $\mathrm{Mg}$ is not detected in $\mathrm{XRD}$, there is a significant effect of $\mathrm{Mg}$ contained sintering ambiance on the temperature-dependent resistivity of the sample. The behavior of resistivity with respect to temperature is shown in Fig. 2. The sample annealed in $\mathrm{Mg}$ ambiance
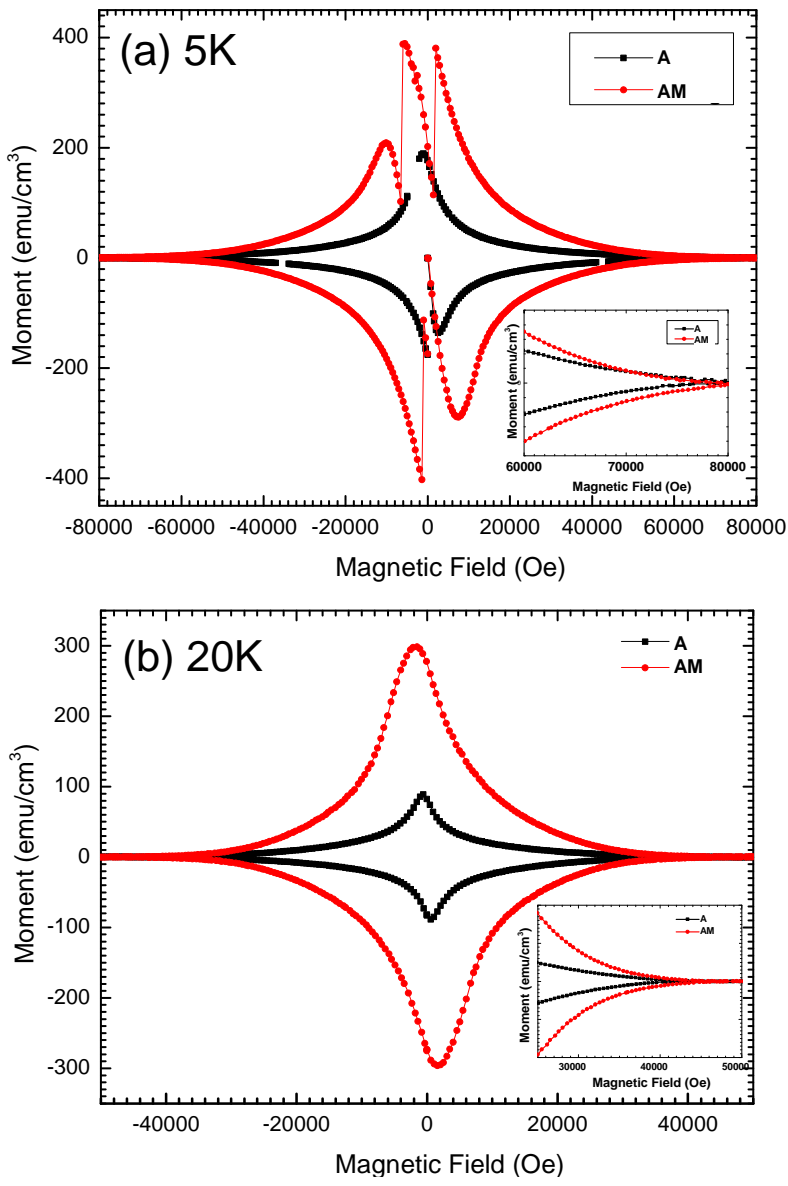

Fig. 3. Magnetization Vs field plot for samples at (a) $5 \mathrm{~K}$ and (b) $20 \mathrm{~K}$. 
has an overall decrease in the resistivity behavior. This improved conductivity showcase the enhancement in the sample connectivity without any increase in the broadening of superconducting transition temperature observed at around $39 \mathrm{~K}$ for both the samples. The higher resistance in case of sample A may be due to the resistive grain boundaries possibly from residual boron and $\mathrm{Mg}$ Here the resistivity for sample AM shows more temperature dependence of electron-phonon interaction than sample A. The Rowell's analysis for the area fraction was carried out for these samples shows an increase in current-carrying-area-fraction $\left(\mathrm{A}_{\mathrm{f}}\right)$ from $\mathrm{A}_{\mathrm{f}}=0.07$ to $\mathrm{A}_{\mathrm{f}}=0.179$ due to sintering in $\mathrm{Mg}$ ambiance. Hence it can be concluded that there is an increase in overall currentcarrying superconducting area.

\subsection{Critical current density}

The critical current density was calculated from the magnetization plots by using Bean's critical state model [14], for samples at $5 \mathrm{~K}$ and $20 \mathrm{~K}$ are shown in Fig. 4 (a) and (b) respectively. A significant increase in the in-field critical current density for sample AM is observed at $5 \mathrm{~K}$
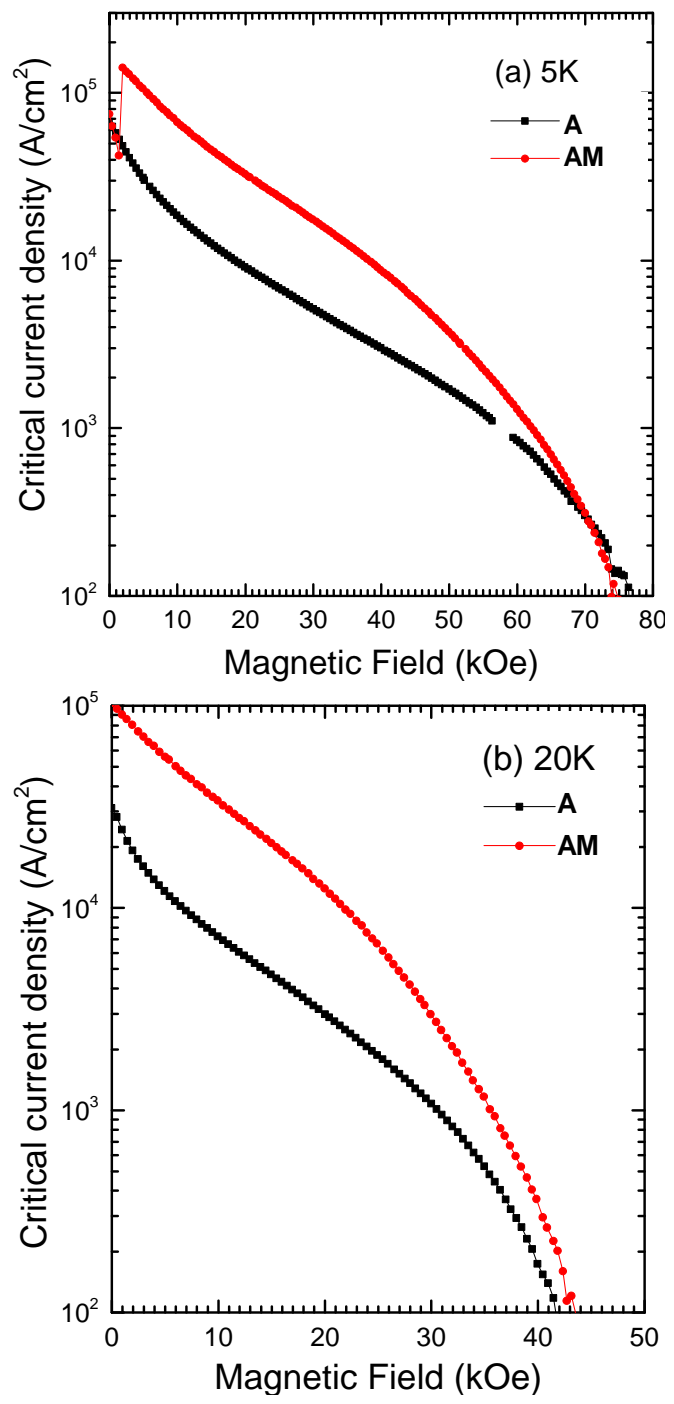

Fig. 4. Critical current density plots for samples at (a) $5 \mathrm{~K}$ and (b) $20 \mathrm{~K}$. as well as $20 \mathrm{~K}$. It is noteworthy to mention that the critical current density values are comparable to those reported by A. Serquis et al [13] for the samples synthesized by hot isostatic pressing technique, meant for high density samples. This improvement in the in-field critical current density strongly supports the argument of increase in the density and in turn the superconducting cross section in the sample due to sintering in $\mathrm{Mg}$ ambiance. This is in line with the resistivity results in which the superconducting transition temperature remains the same and the electron-phonon interaction supports the maximum reduction in the resistivity.

\subsection{Flux pinning properties}

The flux pinning forces were calculated for both the sample and are as shown in Fig. 5. The enhancement in the flux pinning force is clearly depicted from the plots. In order to understand the pinning mechanism in the sample, the reduced flux pinning force plot is shown in the inset. The plot shows a peak at around $H / H_{\text {irr }}$ value of 0.2 which is a typical behavior for the grain boundary pinning mechanism in $\mathrm{MgB}_{2}$. It may be noted here that if excess $\mathrm{Mg}$ would have existed in an unreacted form, it would have formed an effective point defects giving rise to point pinning. No such effect is observed in the sample. This is in support to our XRD plot where no peak for Mg has been detected. Also grain boundary pinning is dominant in $\mathrm{MgB}_{2}$ until effective point defects are created to form a point pinning centers. If $\mathrm{Mg}$ would have just settled itself at the grain boundary, it would have directly affected the resistivity behavior by more or less straightening it. Instead it has shown an enhanced exponential decrease in resistivity driven by electronphonon interaction. Hence it can be inferred that an ambient pressure of $\mathrm{Mg}$ sintering environment has resulted in a self diffusion of $\mathrm{Mg}$ in sample rather than a forced intrusion.

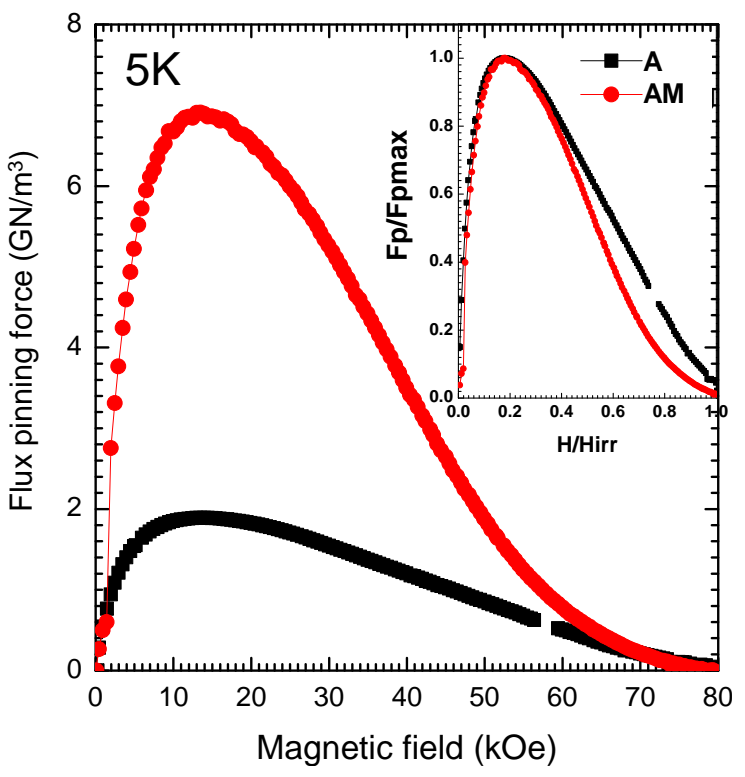

Fig. 5. Flux pinning force plots for samples at $5 \mathrm{~K}$. Inset shows the reduced flux pinning behavior that resembles grain boundary pinning mechanism. 


\section{CONCLUSIONS}

The presence of $\mathrm{Mg}$ vapors at ambient pressure improved the effective superconducting cross sectional area in the sample. The open tube arrangement instead of the sealed tube configuration ensured the self-diffusion of $\mathrm{Mg}$ without creating any excessive $\mathrm{Mg}$ defects in the sample. This in turn resulted in considerable increase in the infield critical current density of the sample.

\section{ACKNOWLEDGMENT}

This work was supported by the Global Research Laboratory Program from the National Research Foundation of Korea (NRF) through a grant funded by the Korean Ministry of Education, Science \& Technology (MEST) (No. 2012-00184).

\section{REFERENCES}

[1] J. Nagamatsu, N. Nakagawa, T. Muranaka, Y. Zenitani, and J. Akimitsu, "Superconductivity at $39 \mathrm{~K}$ in magnesium diboride," Nature, vol. 410, pp. 63, 2001

[2] K.H.P. Kim, J.H. Choi, C.U. Jung, P. Chowdhury, H.S. Lee, M.S Park, H.J. Kim, J.Y. Kim, Z. Du, E.M. Choi, M.S. Kim, W.N Kang, S.I. Lee, G.Y. Sung, J.Y. Lee, "Superconducting properties of well-shaped $\mathrm{MgB}_{2}$ single crystals," Phys. Rev. B, vol. 65, pp.100510, 2002.

[3] A.K. Pradhan, X.Z. Shi, M. Tokunaga, T. Tamegai, Y. Takano, K. Togano, H. Kito, H. Ihara, "Electrical transport and anisotropic superconducting properties in single crystalline and dense polycrystalline $\mathrm{MgB}_{2}$," Phys. Rev. B, vol. 64, pp. 212509, 2001.
[4] A.K. Pradhan, X.Z. Shi, M. Tokunaga, T. Tamegai, Y. Takano, K. Togano, H. Kito, H. Ihara, "Angle-resolved magnetotransport studies in anisotropic $\mathrm{MgB}_{2}$ single crystals," Phys. Rev. B, vol. 65, pp. 144513, 2002.

[5] S. Okuma, S. Togo, and K. Amemori, "Observation of superconductivity in thick amorphous $\mathrm{Mg}_{\mathrm{x}} \mathrm{B}_{1-\mathrm{x}}$ films," Phy. Rev. B, vol. 67 , pp. 172508,2003

[6] B.A. Glowacki, M. Majoros, M. Vickers, J.E. Evetts, Y. Shi and I. McDougall, "Superconductivity of powder-in-tube $\mathrm{MgB}_{2}$ wires," Supercond. Sci. Technol., vol. 14, pp.193, 2001.

[7] J. M. Rowell, "The widely variable resistivity of $\mathrm{MgB}_{2}$ samples," Supercond. Sci. Technol., vol. 16, pp. R17, 2003.

[8] P. A. Sharma, N. Hur, Y. Horibe, C. H. Chen, B. G. Kim, S. Guha M. Z. Cieplak, and S-W. Cheong, "Percolative superconductivity in $\mathrm{Mg}_{1-\mathrm{x}} \mathrm{B}_{2}, "$ Phys. Rev. Lett., vol. 89, pp. 167003, 2002

[9] P. C. Canfield, D. K. Finnemore, S. L. Bud'ko, J. E. Ostenson, G. Lapertot, C. E. Cunningham, and C. Petrovic, "Superconductivity in dense $\mathrm{MgB}_{2}$ wires," Phys. Rev. Lett., vol. 86, pp. 2423, 2001.

[10] Y. Bugoslavsky, L. F. Cohen, G. K. Perkins, M. Polichetti, T. J. Tate, R. Gwilliam, and A. D. Caplin, "Enhancement of the high magnetic field critical current density of superconducting $\mathrm{MgB}_{2}$ by proton irradiation," Nature, vol. 411, pp. 561, 2001.

[11] D. C. Larbalestier, L. D. Cooley, M. O. Rikel, A. A. Polyanskii, J. Jiang, S. Patnaik, X. Y. Cai, D. M. Feldmann, A. Gurevich, and A. A. Squitieri, "Strongly linked current flow in polycrystalline forms of the superconductor $\mathrm{MgB}_{2}$," Nature, vol. 410, pp. 186, 2001.

[12] T. C. Shields, K. Kawano, D. Holdom, and J. S. Abell, "Microstructure and superconducting properties of hot isostatically pressed $\mathrm{MgB}_{2}$," Supercond. Sci. Technol., vol. 15, pp. 202, 2002.

[13] A. Serquis, X. Z. Liao, Y. T. Zhu, J. Y. Coulter, J. Y. Huang, J. O. Willis, D. E. Peterson, F. M. Mueller, N. O. Moreno, J. D. Thompson, V. F. Nesterenko, and S. S. Indrakanti, "Influence of microstructures and crystalline defects on the superconductivity of $\mathrm{MgB}_{2}$, , J. Appl. Phys., vol. 92, pp. 351, 2002.

[14] C.P. Bean, "Magnetization of hard superconductors," Phys. Rev. Lett., vol. 8, pp. 250, 1962. 